\title{
平面分离双栅金属氧化物半导体场效应 晶体管
}

肖德元*，陈国庆，李若加，刘永，沈其昌

中芯国际集成电路制造(上海)有限公司技术发展中心，上海 201203

*E-mail: deyuan_xiao@smics.com

收稿日期: 2006-10-30; 接受日期: 2007-04-10

摘要首次提出并制作了一种全新的平面分离双栅金属氧化物半导体场 效应晶体管, 该器件垂直于沟道方向的电场 $E_{\mathrm{Z}}$ 为一非均匀场. 理论计算, TCAD 三维器件仿真以及实验结果均表明, 通过改变该器件其中任何一个栅 极偏置电压, 能够得到可以调节的输出特性(增益系数)及转移特性曲线, 可 以很方便地调节器件的阈值电压及亚阈值摆幅, 并具备低功耗特点. 这为电

关键词 新颖器件 场效应晶体管 平面分离双栅 亚阈值摆幅可调 路的设计及器件制作提供了更多的灵活性, 既可以简化电路的设计, 又可以 降低MOS 集成电路制造工艺的复杂程度. 平面分离双栅金属氧化物半导体 场效应晶体管制作工艺与目前常规的 CMOS工艺完全兼容.

基于竞争的需要, 一些领先的半导体厂商在缩小芯片尺寸方面不遗余力. 整个半导体工 业一直都在努力满足新型晶体管开发设计中不断增加的挑战, 这些设计要求晶体管的体积更 小、性能更好, 但却要求最小限度地偏离当今半导体工业中标准的制造工艺来生产. 自从 1960 年第 1 个实用的金属氧化物半导体场效应管(MOSFET)在美国Bell实验室被发明以来, 器件的 尺寸已大幅度缩减, 然而第 1 个MOSFET所采用的基本结构并没有发生变化. 当今MOSFET器 件尺寸按比例继续缩小所面临的严重挑战是如何控制器件的漏电流(Ioff)而同时保持较高的电 流驱动能力 (Ion) $)^{[1,2]}$. 短沟道效应在所有的常规金属氧化物半导体场效应晶体管(MOSFET)中 都是常见的. 透过提高沟道区掺杂, 可以降低短沟道效应, 但其代价是电子迁移率降低、速度 减慢、发生电子雪崩击穿的危险加大. 为了维持对MOSFET短沟道的栅控能力, 栅极介质的厚 度也必须减少. 一个理想的MOSFET场效应管, 它基本上是一个仅受栅电压控制的开关, 而短 沟道效应则反映了当沟道长度减小时, 漏极电压对沟道静电场的负面影响. 减小电源电压 $V_{\mathrm{D}}$ 进而降低电源工作功率是器件与工艺的基本选择. 这么做将降低器件的电流, 导致它对器件 以及互连的快速驱动能力的下降. 而电流可以通过减小器件的阈值电压 $V_{\mathrm{T}}$ 及减薄栅氧化层厚 
度来提升, 但这些措施将增加器件的亚阈漏电及栅极的漏电流. 这就是器件物理与工艺所面 临的速度/功率困境 ${ }^{[2]}$. 一种解决方案是采用全耗尽设计 ${ }^{[3]}$. Freescale公司的 MIGFET $^{[4]}$, 三星公 司的 $\Omega$-FinFET ${ }^{[5]}$ 以及Intell公司的Tri-gate FinFET ${ }^{[6]}$ 可以作为未来MOS晶体管的一种选择. 虽 然这些器件均能较好地控制短沟道效应, 但是由于这些器件均属于垂直结构, 故制作工艺较 复杂.

目前工业界在芯片设计制造上普遍采用多项國值电压 $V_{\mathrm{T}}$ 和多项电源电压 $V_{\mathrm{D}}$ 以及比较积 极的电路设计实现电源管理, 以满足电路对速度和功率的不同要求. 以 $0.18 \mu \mathrm{m}$ 逻辑电路为例, 在逻辑处理单元电路部分, 一般采用 $1.8 \mathrm{~V}$ 电源电压, 饱和电流为 $600 \mu \mathrm{A} / \mu \mathrm{m}$, 漏电流为 30 $\mathrm{pA} / \mu \mathrm{m}$, 阈值电压 $V_{\mathrm{T}}$ 为 $0.4 \mathrm{~V}$ 左右, 沟道长度为 $0.18 \mu \mathrm{m}$, 栅氧化层厚度一般为 $3.2 \mathrm{~nm}$; 在外 围输入输出单元电路部分, 一般采用 $3.3 \mathrm{~V}$ 电源电压, 饱和电流为 $600 \mu \mathrm{A} / \mu \mathrm{m}$, 漏电流为 1 $\mathrm{pA} / \mu \mathrm{m}$, 阈值电压 $V_{\mathrm{T}}$ 为 $0.7 \mathrm{~V}$ 左右, 沟道长度为 $0.35 \mu \mathrm{m}$, 栅氧化层厚度一般为 $60 \mathrm{~nm}$. 制造工 艺还是很复杂. 甚至于在 $90 \mathrm{~nm}$ 逻辑电路中还要采用 $1.0,1.8$ 和 $2.5 \mathrm{~V}$ 三种电源电压, 制造工 艺就更复杂. 我们提出的平面分离双栅金属氧化物半导体场效应晶体管, 其阈值电压及亚阈 摆幅(S)可以通过分离双栅中的任意一个来进行调制并具备低功耗特点, 既可以适应芯片对速 度和功率的不同要求, 简化电路设计, 又可以简化制作工艺, 是一种较好的器件结构设计, 它 在系统芯片(SOC)上将得到应用.

\section{1 平面分离双栅场效应管器件结构}

美国专利 USP3999210 “FET having a linear impedance characteristic over a wide range of frequency”(发明人:Yamada, Takaaki)公开了一种沿沟道(源漏)方向的双栅电阻-氧化物-半导体 场效应晶体管. 该器件的栅极由多晶硅电阻材料组成, 在沿沟道(源漏)方向的栅极两端分别引 入电极, 工作时, 此两电极加不同的偏置电压, 在沟道内形成一沿沟道(源漏)方向变化的调制 沟道电导的电场, 以达到在宽频范围具有线性阻抗及高频特性. 中国专利 CN86101937 “电阻氧化物-半导体场效晶体管” (发明人: 叶安祚) 公开了一种绝缘栅型场效应晶体管, 其栅极由电 阻材料构成. 在电阻栅极垂直于源漏连线(沟道)方向的端线的两端制作了两个欧姆接触电极 作为双栅端, 其结构和功能上是等效的. 该器件具有不截止、遥截止或锐截止转移特性, 可根 据需要获得所需的截止电压值和跨导值, 两个栅端可同时作为控制栅和信号栅使用. 该器件 的应用扩大了场效应管的应用范围, 可使电路得到简化, 能有效地解决大信号堵塞、自动增益 控制动态范围窄等问题.

但是以上两种器件, 由于其栅极均由电阻材料构成, 且在电阻栅极两端制作了两个欧姆 接触电极作为双栅端, 器件工作时加不同的偏置电压, 该电阻栅将消耗掉一定的功率, 在低功 率应用场合将受到限制.

本文首次提出一种全新的平面分离双栅金属氧化物半导体场效应晶体管(planar split dual gate metal-oxide-semiconductor field-effect transistor, 简称 PSDG MOSFET), 它是由 3 个 MOS 二极管与两个背靠背相接的 PN 结所构成, 图 1 所示为一 $\mathrm{N}$ 型沟道平面分离双栅金属氧化物半 导体场效应晶体管(PSDG MOSFET)的透视图. 由一个有两个 N+区域(即源极与漏极)的 $\mathrm{p}$ 型半 导体所组成. 栅极在平面从物理及电性上被分离开并由绝缘介质所填充形成两个栅极, 称之 
为前栅(GF, 或 G1) 与后栅(GB, 或 G2), 因而它是一个五端点器件. 事实上, 柱被分割成 3 部分: 前栅(GF)上栅偏压为 $V_{\mathrm{GF}}$, 后栅(GF) 上栅偏压为 $V_{\mathrm{GB}}$, 中间被分割部分其栅偏压为一非均匀、受 前栅 $V_{\mathrm{GF}}$ 与后栅 $V_{\mathrm{GB}}$ 影响, 缓慢变化的电压. 即平面分离双栅金属氧化物半导体场效应晶体管 由前后两个加不同栅偏压 $V_{\mathrm{GF}}$ 与 $V_{\mathrm{GB}}$ 、中间由无数个处于不同栅偏压的微 MOS 晶体管所构成. 垂直于沟道方向, 电场 $E_{\mathrm{Z}}$ 为一非均匀场.

平面分离双栅金属氧化物半导体场效应晶体管版图设计由图 2 所示. 器件的基本参数是 沟道长度 $L$, 沟道宽度 $W$, 其中沟道宽度被分割成 $Z_{1}, Z_{2}$ 和 $Z_{3}$ 三部分, 绝缘体厚度为 $d$, 祄底掺 杂浓度为 $N_{\mathrm{A}}$.

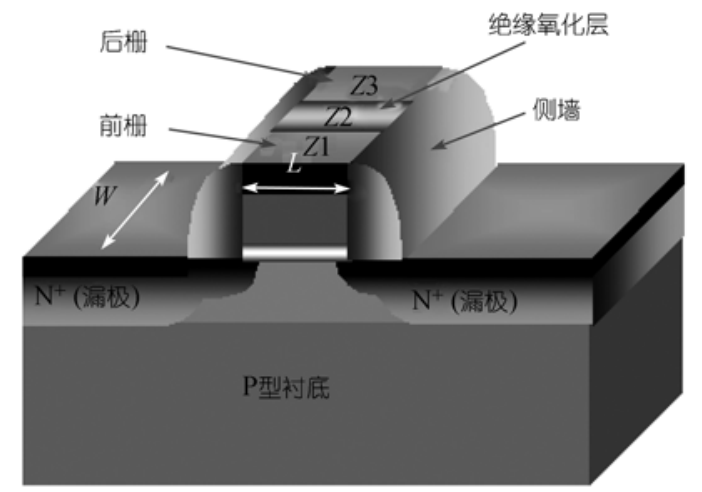

图 $1 \quad \mathrm{~N}$ 型沟道垂直于沟道(源漏)方向的平面分离 双栅场效应管透视图

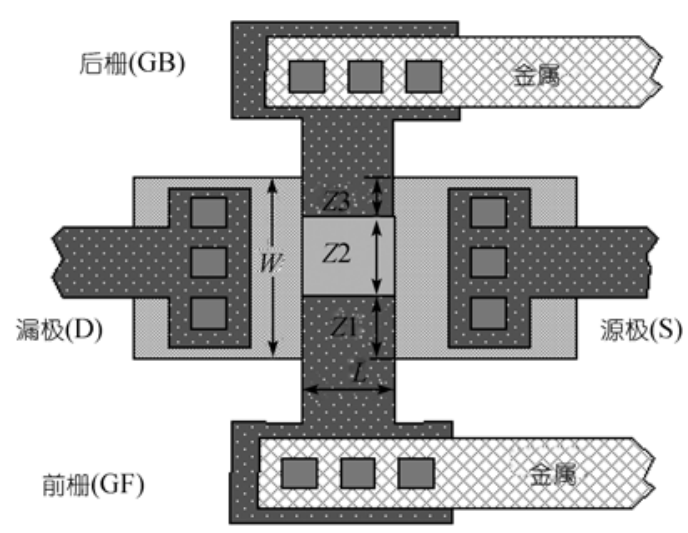

图 2 平面分离双柱金属氧化物半导体场效应晶体 管版面设计图

\section{2 平面分离双栅场效应管三维能带图}

在这里, 我们定义源接触作为电压的基准. 当栅上不加电压时, 源极与漏极相当于两个背 靠背相接的 PN 结, 能从源极流到漏极的唯一电流是反向漏电流. 当栅上加足够大的正偏压使 得两个 $\mathrm{N}^{+}$区域(即源极与漏极)之间形成表面反型层(或反型沟道)时, 源极与漏极就靠这一导电 的表面 $\mathrm{N}$ 型沟道连结起来, 在沟道內能流过大的电流. 现在, 由于有了两个栅极, 因而通过改 变两个栅极电压可以很灵活地调制此沟道的电导. 当然, 祄底也可以有基准电压, 或处于反向 偏置状态; 此祄底反向偏置也会影响沟道电导.

图 3 给出平面分离双栅金属氧化物半导体场效应晶体管处于不同偏置电压状态下的三维 能带图(3-D energy band diagram). 当源漏接触两端加一偏置电压时, MOS 结构处于非平衡状 态. 由于栅被分割成为 3 部分：前栅(GF, 或 G1)上栅偏压为 $V_{\mathrm{GF}}$, 后椓(GB, 或 G2)上栅偏压为 $V_{\mathrm{GB}}$, 中间被分割部分其栅偏压为一非均匀、受前栅 $V_{\mathrm{GF}}$ 与后栅 $V_{\mathrm{GB}}$ 影响而渐变的电压, 此时的 能带将是扭曲的. 因此, 从能带图角度上看, 我们提出的这种平面分离双椓金属氧化物半导体 场效应晶体管是一种三维 MOS 器件, 其他如双栅 FinFET 和 Omega FinFET 以及三栅 FinFET 场效应晶体管均为二维 MOS 器件. 


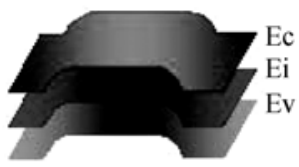

(a)

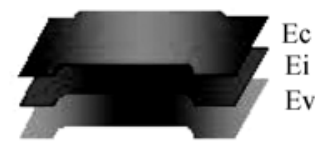

(b)

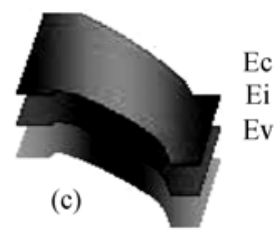

(c)

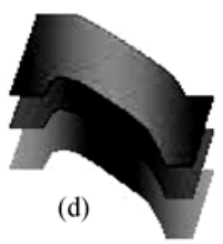

图 3 平面分离双栅晶体管三维能带图

\section{3 平面分离双栅场效应管电流-电压特性}

在下列理想条件下 $[7]$ :

1. 栅结构为一理想 MOS 二极管, 即无界面陷阱, 固定氧化层电荷或功函数差;

2. 仅考虑漂移电流;

3. 反型层中载流子的迁移率为常数;

4. 沟道内杂质浓度为均匀分布;

5. 反向漏电流可忽略;

6. 沟道中由栅电压所产生的横向电场远大于由漏电压所产生的纵向电场. 此条件为缓变 沟道近似法, 通常可适用于长沟道 MOSFET. 基于此种近似法, 祄底表面耗尽区中所包含的电 荷量仅由栅电压所产生的电场感应所生成.

此外, 在这里由于平面分离双栅的缘故, 使得栅电压所产生的沿垂直于沟道电流方向的 横向电场不均匀. 该电场受前栅偏城(VGF)、后栅偏压(VGB)以及中间分割部分(受前栅与后栅 偏压影响)栅偏压所影响. 据此, 我们推导出基本的平面分离双栅金属氧化物半导体场效应晶 体管电流-电压特性方程为

$$
\begin{aligned}
I_{\mathrm{D}}= & \frac{Z_{1}}{L} \mu_{n} C_{0}\left\{\left(V_{\mathrm{GF}}-2 \psi_{\mathrm{B}}-\frac{V_{\mathrm{D}}}{2}\right) V_{\mathrm{D}}-\frac{2}{3} \frac{\sqrt{2 \varepsilon_{\mathrm{s}} e N_{\mathrm{A}}}}{C_{0}}\left[\left(V_{\mathrm{D}}+2 \psi_{\mathrm{B}}\right)^{3 / 2}-\left(2 \psi_{\mathrm{B}}\right)^{3 / 2}\right]\right\} \\
& +\frac{Z_{2}}{L} \mu_{n} C_{0}\left\{\frac{1}{2} V_{\mathrm{D}}\left(V_{\mathrm{GB}}+V_{\mathrm{GF}}\right)-\left(2 \psi_{\mathrm{B}}+\frac{V_{\mathrm{D}}}{2}\right) V_{\mathrm{D}}-\frac{2}{3} \frac{\sqrt{2 \varepsilon_{\mathrm{s}} e N_{\mathrm{A}}}}{C_{0}}\left[\left(V_{\mathrm{D}}+2 \psi_{\mathrm{B}}\right)^{3 / 2}-\left(2 \psi_{\mathrm{B}}\right)^{3 / 2}\right]\right\} \\
& +\frac{Z_{3}}{L} \mu_{n} C_{0}\left\{\left(V_{\mathrm{GB}}-2 \psi_{\mathrm{B}}-\frac{V_{\mathrm{D}}}{2}\right) V_{D}-\frac{2}{3} \frac{\sqrt{2 \varepsilon_{\mathrm{s}} e N_{\mathrm{A}}}}{C_{0}}\left[\left(V_{\mathrm{D}}+2 \psi_{\mathrm{B}}\right)^{3 / 2}-\left(2 \psi_{\mathrm{B}}\right)^{3 / 2}\right]\right\},
\end{aligned}
$$

式中 $e$ 为元电荷, $\mu_{n}$ 为电子迁移率, $\varepsilon_{0 \mathrm{x}}$ 为绝缘体电容率, $\varepsilon_{\mathrm{s}}$ 为半导体电容率, $\psi_{\mathrm{B}}$ 为半导体的 Shottkey势垒高度, $d$ 为绝缘体厚度, $N_{\mathrm{A}}$ 为祄底掺杂浓度, $C_{0}=\varepsilon_{0 x} / d$ 为每单位面积的栅极电容. 考 虑对称平面分离双栅情形, 即前栅极与后栅极宽相等 $\left(Z_{1}=Z_{3}, Z_{1}+Z_{2}+Z_{3}=W\right)$, 上式简化为

$$
I_{\mathrm{D}}=\frac{W}{L} \mu_{n} C_{0}\left\{\frac{1}{2} V_{\mathrm{D}}\left(V_{\mathrm{GB}}+V_{\mathrm{GF}}\right)-\left(2 \psi_{\mathrm{B}}+\frac{V_{\mathrm{D}}}{2}\right) V_{\mathrm{D}}-\frac{2}{3} \frac{\sqrt{2 \varepsilon_{\mathrm{s}} e N_{\mathrm{A}}}}{C_{0}}\left[\left(V_{\mathrm{D}}+2 \psi_{\mathrm{B}}\right)^{3 / 2}-\left(2 \psi_{\mathrm{B}}\right)^{3 / 2}\right]\right\} \text {. }
$$

即在对称平面分离双栅情形下, 总漏极电流 $I_{\mathrm{D}}$ 仅与总的栅宽 $W$ 成正比例, 而与中间分离 宽度 $Z_{2}$ 无关, 此时的平面分离双栅晶体管相当于栅宽 $W$, 沟道长度 $L$, 工作电压 $V_{\mathrm{D}}$, 栅平均电 压为 $\left(V_{\mathrm{GF}}+V_{\mathrm{GB}}\right) / 2$ 的 $\mathrm{MOS}$ 晶体管, 因而通过改变其中任何一个栅极电压可以很灵活地调制此沟 道的电导, 也即总漏极电流 $I_{\mathrm{D}}$. 因而该器件将具备阈值电压可调特性. 


\section{4 平面分离双栅场效应管亚阈值特性}

当栅电压小于國值电压，且半导体表面只有弱反型时，其对应的漏电流称为亚阈值电流. 因为亚阈值区描述器件如何开启与关闭, 所以当 MOS 场效应晶体管用来作为在数字逻辑开关 电路与存储器应用上的低电压与低功率器件使用时, 器件的亚阈值特性显得特别重要. 在亚 阈值区域内, 漏极电流扩散并非漂移所主导. 为简单起见, 我们考虑平面对称分离双栅情形, 即 $Z_{1}=Z_{2}=Z_{3}=W / 3$, 得到单位面积漏极电流密度为

$$
\begin{aligned}
J_{\mathrm{D}}= & \frac{e D_{n} n_{i} \exp \left(-\beta \psi_{\mathrm{B}}\right)}{3 L}\left(1-e^{-\beta V_{\mathrm{D}}}\right)\left[\left(e^{\beta\left(V_{\mathrm{GF}}-V_{\mathrm{T}}\right)}+e^{\beta\left(V_{\mathrm{GB}}-V_{\mathrm{T}}\right)}\right)\right. \\
& \left.+\frac{1}{\beta\left(V_{\mathrm{GB}}-V_{\mathrm{GF}}\right)}\left(e^{\beta\left(V_{\mathrm{GB}}-V_{\mathrm{T}}\right)}-e^{\beta\left(V_{\mathrm{GF}}-V_{\mathrm{T}}\right)}\right)\right],
\end{aligned}
$$

其中, $\beta=e / k T$. 亚阈值摆幅 $(S)$ 定义为 $\left[\partial\left(\lg I_{\mathrm{D}}\right) / \partial V_{\mathrm{G}}\right]^{-1}$, 根据(3)式计算得出的平面分离双栅金 属氧化物半导体场效应晶体管亚阈值摆幅 $(S)$ 将随任一栅电压变化而变化. 也就是说, 平面分 离双栅晶体管亚阈值特性可以通过分离双栅中的任意一个来进行调制. 理论上, 还可以得到 最小的亚阈值摆幅 $(S)$ 为 $75 \mathrm{mV} / \mathrm{dec}$.

\section{5 平面分离双栅场效应管三维器件仿真}

Pei等人采用器件仿真工具进行垂直分离双栅场效应管器件三维仿真 ${ }^{[8]}$. 我们采用SYNOPSYS(新思科技)公司的三维器件 3D Devise和 3D Dessis仿真工具进行器件的三维仿真. 图 4 为模 拟得到的典型的平面分离双栅金属氧化物半导体场效应晶体管亚阈值特性曲线. 从图上同样可 以看出, 在亚阈区, 漏极电流密度与 $V_{\mathrm{GF}}$ 呈指数关系. 当 $V_{\mathrm{GF}}$ 为负偏压时, Ioff可以降低至接近传统 MOS场效应晶体管的三分之一, 这与我们的计算结果相一致. 图 5 为模拟得到的典型的平面分 离双栅金属氧化物半导体场效应晶体管亚阈值摆幅 $(S)$ 随一栅电压 $V_{\mathrm{GB}}\left(V_{\mathrm{G} 2}\right)$ 变化的曲线.

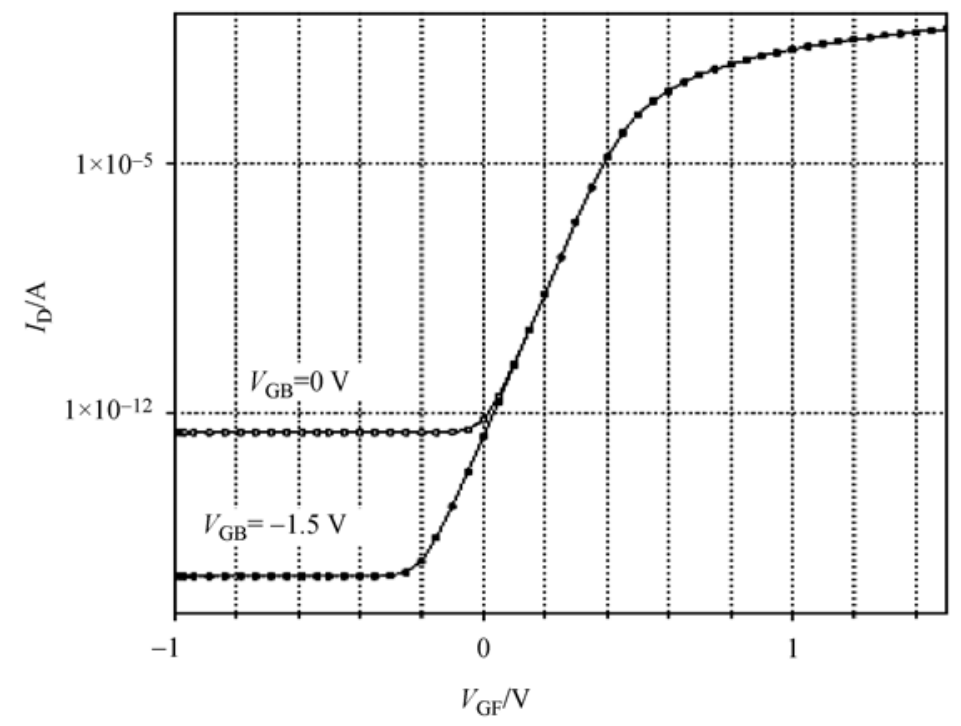

图 4 TCAD 模拟得到的典型的平面分离双栅场效应晶体管亚阈值特性曲线 其中栅极分离宽度 $Z 2=1 / 3 W, W / L=10 / 0.18$ 


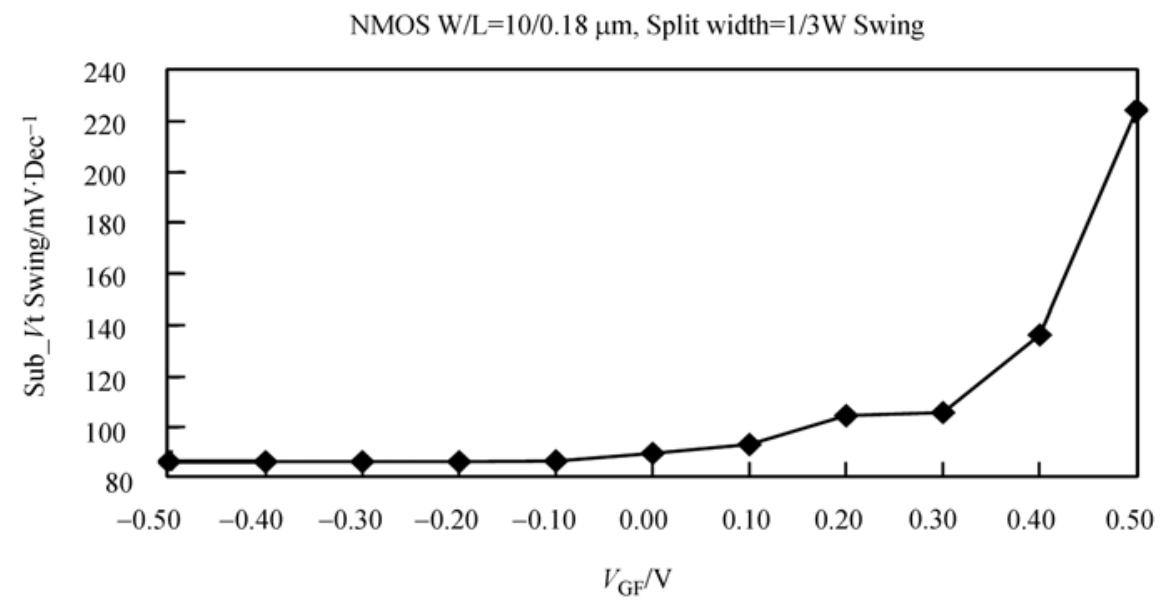

图 5 TCAD 模拟得到的典型的平面分离双栅金属氧化物半导体场效应晶体管亚阈值摆幅 $(S)$ 随一栅电 压 $V_{\mathrm{GF}}$ 变化曲线

\section{6 平面分离双栅场效应管三维器件制作}

CMOS 技术在工艺家族中最有代表性, 我们采用与常规 $0.18 \mu \mathrm{m}$ CMOS 集成电路制造兼 容的设计规则和工艺流程来制作平面分离双栅晶体管(PSDG MOSFET)器件. 其主要制造流程 模块如下:

1. 浅槽隔离有源区形成工艺(shallow trench isolation, active area formation);

2. 双阱工艺(dual P well and $\mathrm{N}$ well implantation);

3. 多晶硅栅结构工艺(gate formation);

4. 轻漏掺杂 (LDD)注入工艺(NLDD, PLDD implantation);

5. 侧墙的形成(spacer formation);

6. 自对准源/漏(S/D)注入工艺 $\left(\mathrm{N}^{+} / \mathrm{P}^{+}\right.$self aligned source and drain implantation);

7. 平面分离双栅形成(planar split dual gate formation);

8. 金属硅化物工艺(co silicide process);

9. 接触孔的形成与互连工艺(contact formation and interconnect);

10. 表面针化工艺(passivation);

11. 参数测试(wafer acceptance test).

在完成好第 6 流程模块自对准源/漏(S/D)注入工艺后, 利用深紫外线光刻技术刻印平面分 离多晶硅双栅的精细结构, 分两步进行平面分离双栅多晶硅刻蚀. 先采用等离子体蚀刻工艺 刻蚀多晶硅栅, 然后采用湿法腐刻工艺刻蚀多晶硅栅得到平面垂直分离的多晶硅双栅结构. 之后完全按照常规 $0.18 \mu \mathrm{m}$ CMOS 集成电路制造工艺流程完成剩余的工艺流程. 图 6 为平面分 离双栅晶体管沿垂直于沟道(宽度)方向结构透射电镜(TEM)照片, 从图片上可以看出, 栅极分 离 $Z_{2}$ 部分的多晶硅已全部去除且为二氧化硅绝缘层所填充. 图 7 为平面分离双栅晶体管沿沟 道方向结构扫描电镜照片. 


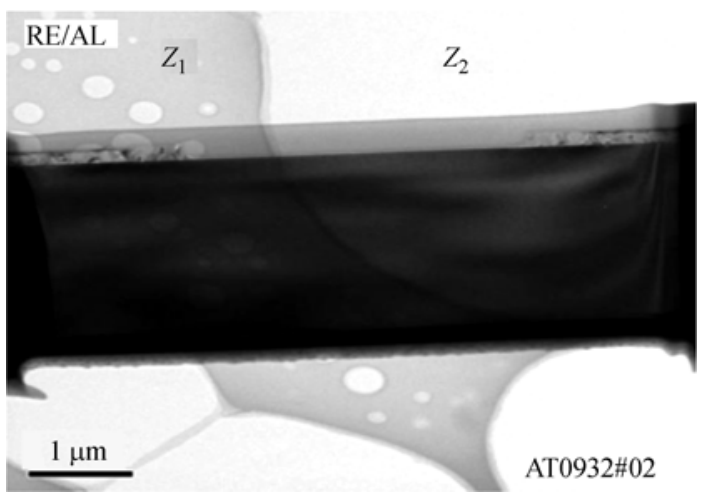

图 6 平面分离双栅晶体管沿垂直于沟道(宽度)方 向结构透射电镜(TEM)照片

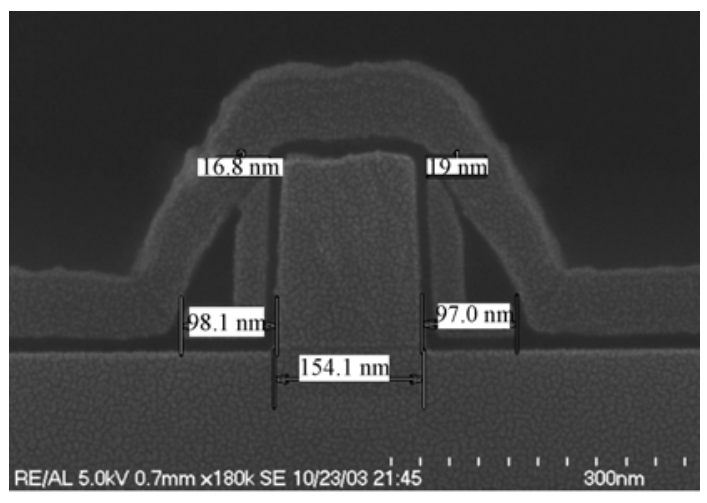

图 7 平面分离双栅晶体管沿沟道方向结构扫描电 镜照片

\section{7 讨论和结论}

对制作的平面分离双栅晶体管(Lot Id: AT0932)进行了器件特性参数测试. 实验结果与理 论计算及 TCAD 模拟符合得较好.

图 8 为实验测量得到的平面分离双栅晶体管漏极输出特性曲线. 比较左右图输出特性曲 线可以看出, 在不同的 $V_{\mathrm{GB}}$ 偏置条件下, 我们可以得到不同的输出特性曲线, 也即可以对器件 的增益系数进行调制.

图 9 为实验测量得到的平面分离双栅晶体管转移特性曲线. 通过改变其中任何一个栅极 电压可以很灵活地调制该器件沟道电导, 也即总漏极电流 $I_{\mathrm{D}}$. 证实该器件将具备阈值电压可调 特性. 目前的平面工艺需要制作多个 MOS 晶体管, 每个晶体管具有不同的栅氧化层厚度及不 同的掺杂浓度以获得不同的阈值电压. 而采用平面分离双栅晶体管设计制造工艺, 在不同的 $V_{\mathrm{GB}}$ 偏置下, 我们可以得到一系列的转移特性曲线, 通过改变其中任何一个栅极偏置电压可以 很方便地调节器件的阈值电压并具备低功耗特点, 我们将只需要一种晶体管, 这样既可以简 化电路的设计又可以降低 MOS 集成电路制造工艺的复杂程度, 这为电路的设计及器件制作提 供了更多的灵活性.

图 10 为实验测量得到栅极分离宽度为 $Z_{2}=W / 3$ 的平面分离双栅晶体管转移特性曲线(取对 数坐标).

图 11 为实验测量得到的平面分离双栅晶体管亚阈值摆幅 $(S)$ 随一栅电压 $V_{\mathrm{GB}}$ 变化曲线, $S$ 随栅电压 $V_{\mathrm{GB}}$ 增加而变大. 这与我们的理论计算和 TCAD 模拟结果相吻合. 通过改变其中任何 一个栅极偏置电压可以很方便地调节器件的阈值电压及亚阈摆幅, 这为电路的设计及器件制 作提供了更多的灵活性.

我们首次提出并制作了一种全新的平面分离双栅金属氧化物半导体场效应晶体管, 该器 件垂直于沟道方向的电场 $E_{\mathrm{Z}}$ 为一非均匀场. 理论计算和 TCAD 三维器件仿真以及实验结果均 表明, 通过改变该器件其中任何一个栅极偏置电压, 能够得到可以调节的输出特性(增益系数) 


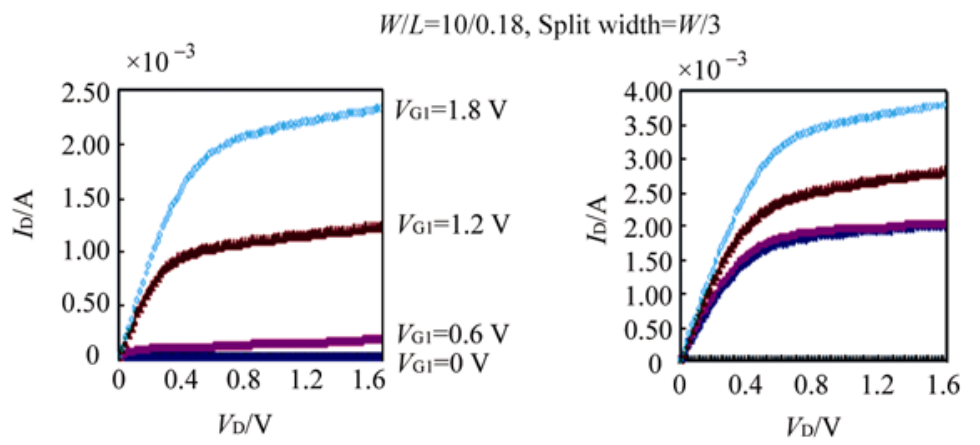

图 8 实验测量得到的栅极分离宽度 $Z 2=1 / 3 W$ 晶体管漏极输出特性曲线

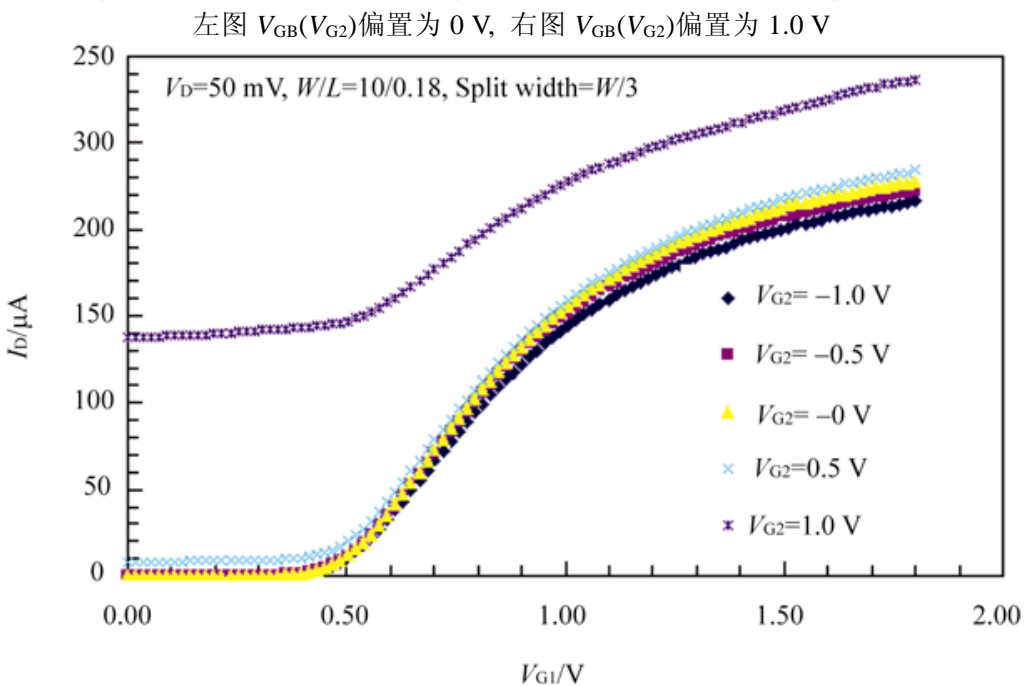

图 9 实验测量得到的平面分离双栅晶体管转移特性曲线 其中栅极分离宽度 $Z 2=1 / 3 W$, 漏极电压 $V_{\mathrm{D}}$ 为 $50 \mathrm{mV}$

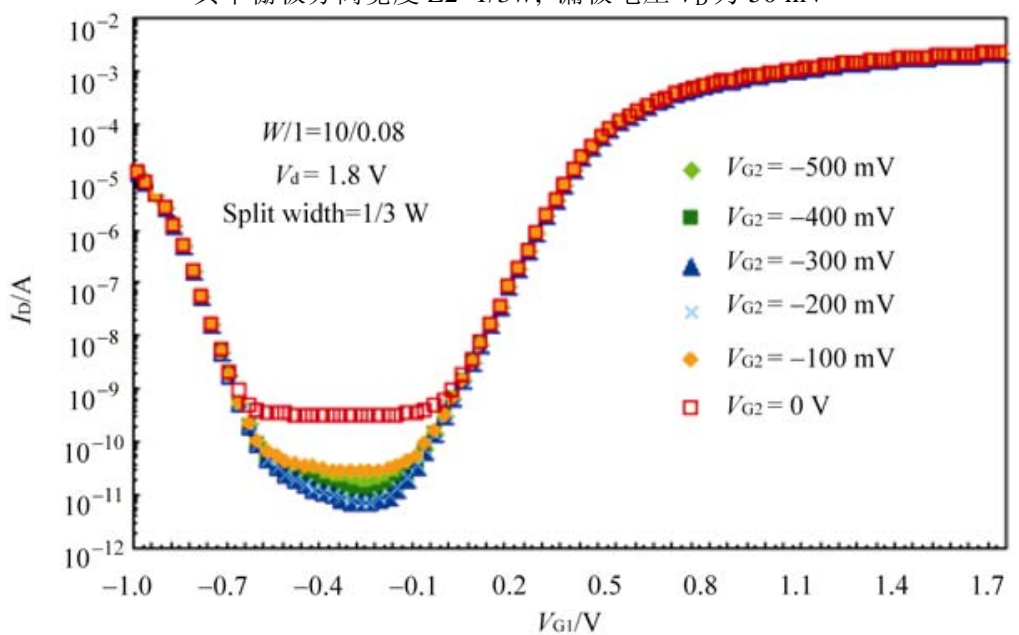

图 10 实验测量得到的平面分离双栅晶体管转移特性曲线(取对数坐标)

栅极分离宽度 $Z_{2}=W / 3$, 漏极电压 $V_{\mathrm{D}}$ 为 $1.8 \mathrm{~V}$; 当另一栅极偏置为 $-200 \mathrm{mV}$ 时, 得到亚阈值摆幅 $(S)$ 为 $80 \mathrm{mV} / \mathrm{dec}$. 其关 态漏电流 Ioff 为 $16.9 \mathrm{pA} / \mu \mathrm{m}$, 饱和电流 Idsat 为 $230 \mu \mathrm{A} / \mu \mathrm{m}$ 


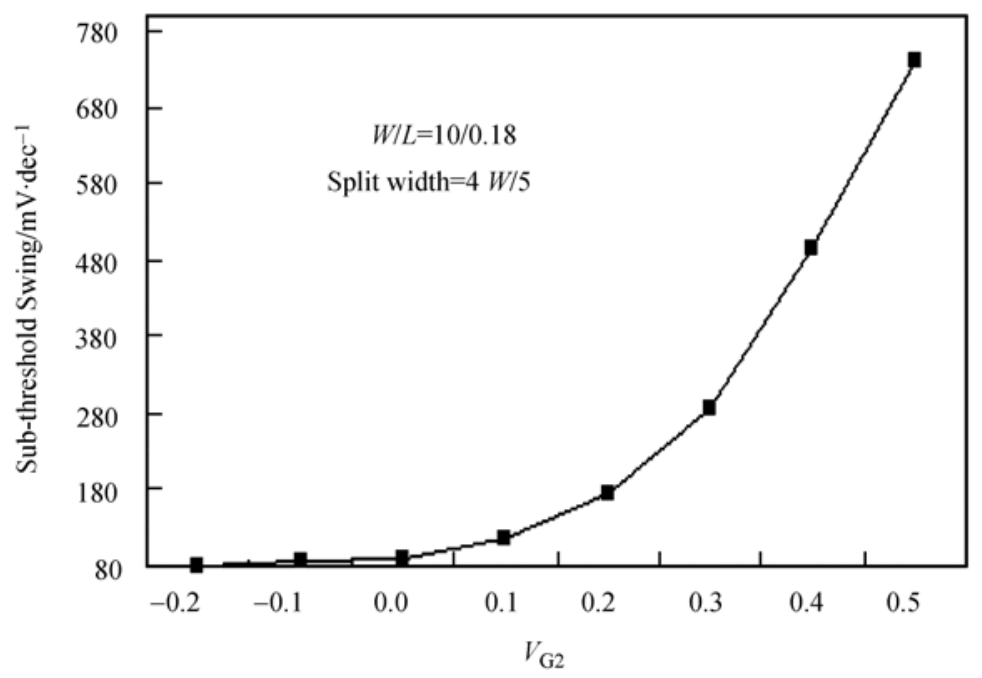

图 11 实验测量得到的平面分离双栅晶体管亚阈值摆幅 $(S)$ 随一栅电压 $V_{\mathrm{GB}}\left(V_{\mathrm{G} 2}\right)$ 变化曲线 棚极分离宽度 $Z_{2}=4 W / 5$

及转移特性曲线, 可以很方便地调节器件的阈值电压及亚阈值摆幅并具备低功耗特点, 这为 电路的设计及器件制作提供了更多的灵活性, 既可以简化电路的设计又可以降低 MOS 集成电 路制造工艺的复杂程度. 感谢新思科技(SYSNOPSYS)袁海江和中芯国际杨勇胜在 3D 三维器件仿真方面及卢 普生、陈良成、洪中山、杨芸、严祥成以及三重野文健等在器件制作方面所给予的 帮助.

\section{参考文献}

1 ITRS. PIDS section, Table 2a, 2001. Http://www.itrs.net/Links/2001ITRS/PIDS.pdf

2 Hu C. Device challenges and opportunities. In: Symposium on VLSI Technology Digest of Technical Papers. Kyoto: The Japan Society of Applied Physics, 2004. 4-5

3 Chau, R, Kavalieros J, Roberds B, et al. $30 \mathrm{~nm}$ physical gate length CMOS transistors with 1.0 ps n-MOS and 1.7 ps p-MOS gate delays. In: International Electron Devices Meeting, IEDM. San Francisco: IEEE Press, 2000. $45-48$

4 Mathew L, Yang D, Kalpat S, et al. Multiple independent gate field effect transistor (MIGFET)-multi-Fin RF mixer architecture, three independent gates (MIGFET-T) operation and temperature characteristics. In: Symposium on VLSI Technology Digest of Technical Papers. Kyoto: The Japan Society of Applied Physics, 2005. 200201

5 Park T, Choi S, Lee D H, et al. Fabrication of body-tied FinFETs (Omega MOSFETs) using bulk Si wafers. In: 
Symposium on VLSI Technology Digest of Technical Papers. Kyoto: The Japan Society of Applied Physics, 2003. $135-136$

6 Doyle B, Boyanov B, Datta S, et al. Tri-gate fully-depleted CMOS transistors: fabrication, design and layout. In: Symposium on VLSI Technology Digest of Technical Papers. Kyoto: The Japan Society of Applied Physics, 2003. $133-134$

7 Sze S M. Semiconductor Devices. New York: John Wiley \& Sons Inc, 2002

8 Pei G, Ni W P, Abhishek V K, et al. A physical compact model of DG MOSFET for mixed-signal circuit applications-part I: model description. IEEE Trans Electron Dev, 2003, 50(10): 2135-2143 